\title{
A Syndrome Definition Validation Approach for Ebola Virus Disease
}

\author{
Dino Rumoro ${ }^{1}$, Shital Shah 1 , Marilyn Hallock ${ }^{1}$, Gillian Gibbs*1, Gordon Trenholme ${ }^{1}$ and \\ Michael J. Waddell ${ }^{2}$
}

${ }^{1}$ Emergency Medicine, Rush University Medical Center, Chicago, IL, USA; ${ }^{2}$ Pangaea Information Technologies, Chicago, IL, USA

\section{Objective}

To develop and validate an Ebola virus disease syndrome definition within the GUARDIAN (Geographic Utilization of Artificial Intelligence in Real-Time for Disease Identification and Alert Notification) surveillance system.

\section{Introduction}

The 2014 Ebola outbreak in West Africa is one of the largest Ebola outbreaks in history. Early detection is critical for rapid initiation of treatment, infection control and emergency response plans. To facilitate clinicians' ability to detect Ebola, various syndrome definitions have been developed.

\section{Methods}

To create and validate a detailed syndrome definition for Ebola, we utilized the literature based methodology developed and documented by GUARDIAN researchers. ${ }^{1,2}$ The syndrome definition utilized clinical signs and symptoms that were documented in historical Ebola cases.

A testing sample of 800 randomly selected emergency department cases (i.e., true negative cases) and 200 synthetically generated cases (i.e., true positive cases) was created. These 1,000 sample cases were evaluated by the GUARDIAN surveillance system to determine the probability of matching the Ebola syndrome definition. A probability of $\geq 90 \%$ was utilized to designate positive Ebola cases.

We identified the main signs and symptoms contributing to the identification of Ebola cases and conducted statistical performance metrics. Clinical review of the false positive and false negative cases along with a sample of true positive and true negative cases was conducted by a board certified emergency physician and an infectious diseases expert.

\section{Results}

The Ebola syndrome definition was developed with 14 articles (10 used for developing the syndrome definition, and 4 used for testing the definition). The sample size for these articles was between 1 and 217 positive Ebola cases, with a total of 678 cases across the 14 articles. The publication timeframe for the articles was from 1977 to 2005 . Some of the main signs and symptoms from the historical cases that contribute to the Ebola syndrome definition are presented in Table 1.

The initial results for the sample testing data showed accuracy, sensitivity, and specificity were $96 \%, 89 \%$, and $97 \%$, respectively. There were a total of 22 false negative and 27 false positive cases.

\section{Conclusions}

The initial Ebola syndrome definition utilized by the GUARDIAN surveillance system contains similar signs and symptoms to the current CDC case definition, but also includes additional signs and symptoms such as abdominal tenderness, thrombocytopenia, nausea, malaise, weakness, and loss of appetite. In addition, the GUARDIAN system provides the relative importance of identified signs and symptoms and allows for proactive surveillance of emergency department patients in real-time. Though we didn't include epidemiologic risk factors, such as travel to an infected region or contact with an infected person in the syndrome definition, GUARDIAN has above $89 \%$ sensitivity and specificity. Thus, inclusion of epidemiologic risk factors would further enhance the early detection of Ebola and possibly other viral hemorrhagic fevers when used with the appropriate high risk population.

Table 1. Main signs and symptoms of the Ebola syndrome definition.

\begin{tabular}{|c|c|}
\hline Signs and Symptoms & Relative Importance (\%) \\
\hline Fever* & 98.1 \\
\hline Abdominal Tenderness & 100.0 \\
\hline Malaise & 100.0 \\
\hline Thrombocytopenia & 100.0 \\
\hline Weakness & 80.5 \\
\hline Headache* & 80.3 \\
\hline Diarrhea* & 79.7 \\
\hline Nausea & 72.1 \\
\hline Vomiting* & 67.8 \\
\hline Loss of appetite & 64.9 \\
\hline Abnormal bleeding & 63.0 \\
\hline Abdominal pain* & 61.3 \\
\hline
\end{tabular}

* Signs and symptoms that are included in the Centers for Disease Control and Prevention (CDC) Ebola case definition.

\section{Keywords}

Ebola; GUARDIAN; syndrome definition

\section{Acknowledgments}

GUARDIAN is funded by the US Department of Defense, Telemedicine and Advanced Technology Research Center, Award numbers W81XWH-09-1-0662 and W81XWH-11-1-0711.

\section{References}

1. Silva J, Rumoro D, Hallock M, Shah S, Gibbs G, Waddell M, Thomas K. Disease profile development methodology for syndromic surveillance of biological threat agents. Emerging Health Threats Journal. 2011; 4(11129).

2. Silva J, Shah S, Rumoro D, Hallock M, Gibbs G, Waddell M. A Novel Syndrome Definition Validation Approach for Rarely Occurring Diseases. Online Journal of Public Health Informatics. 2013; 5(1).

*Gillian Gibbs

E-mail: Gillian_Gibbs@rush.edu 